\title{
Six-dimensional calculation of the vibrational spectrum of the HFCO molecule
}

\author{
Alexandra Viel ${ }^{\text {a) }}$ and Claude Leforestier ${ }^{\text {b) }}$ \\ Laboratoire de Structure et Dynamique des Systèmes Moléculaires et Solides (UMR 5636), \\ Université Montpellier 2, Bât. 15 cc 014, 34095 Montpellier Cedex 5, France
}

(Received 10 August 1999; accepted 21 October 1999)

Bound energy levels, up to $5000 \mathrm{~cm}^{-1}$ of internal excitation, have been computed for the HFCO molecule. An exact six-dimensional (6D) quantum Hamiltonian, expressed in terms of Jacobi vectors, has been used. It is shown to lead to a compact form of the kinetic energy operator, easy to implement in the calculations. The primary discrete variable representation (DVR) basis set has been contracted by means of the adiabatic pseudospectral method of Friesner et al. [J. Chem. Phys. 99, 324 (1993)]. Two different, global, potential energy surfaces have been considered. The calculated energy levels have been successfully assigned by an automatic labeling procedure. These levels have been compared to the experimental results, providing a test of the accuracy of the existing surfaces. (C) 2000 American Institute of Physics. [S0021-9606(00)01503-8]

\section{INTRODUCTION}

The spectroscopy of the methyl fluoride molecule HFCO has been the subject of intensive experimental studies conducted by Moore and co-workers. ${ }^{1,2}$ This molecule is of particular interest as it displays a relatively low dissociation threshold, circa $14000 \mathrm{~cm}^{-1}$, as compared to formaldehyde $\left(\sim 28000 \mathrm{~cm}^{-1}\right)$. Using stimulated emission pumping (SEP) experiments, they have been able to observe and characterize energy levels (bright states) in the range 13000-23000 $\mathrm{cm}^{-1}$, which correspond to metastable states displaying predissociation widths. The equilibrium geometry of the $S_{1}$ excited electronic state reached by the pump laser strongly favors highly excited states in the out-of-plane $\mathrm{H}$ bending mode $\left(\nu_{6}\right)$, up to 22 quanta, during the dump step. Moderately excited states of the $\mathrm{CO}$ stretching mode $\left(\nu_{2}\right)$ were also observed.

The main experimental finding concerns the increasing decoupling, with respect to internal energy, of the $\nu_{6}$ mode from the other modes, and in particular from those leading to dissociation into $\mathrm{HF}+\mathrm{CO}$. As a consequence, Moore and co-workers were able to fit the (bright) energy levels to a spectroscopical Hamiltonian involving only the $\nu_{2}$ and $\nu_{6}$ modes,

$$
\begin{aligned}
E_{s p}\left(n_{2}, n_{6}\right)= & \omega_{2}\left(n_{2}+\frac{1}{2}\right)+\omega_{6}\left(n_{6}+\frac{1}{2}\right)+x_{22}\left(n_{2}+\frac{1}{2}\right)^{2} \\
& +x_{26}\left(n_{2}+\frac{1}{2}\right)\left(n_{6}+\frac{1}{2}\right)+x_{66}\left(n_{6}+\frac{1}{2}\right)^{2} \\
& +y_{222}\left(n_{2}+\frac{1}{2}\right)^{3}+\cdots .
\end{aligned}
$$

These studies have stimulated the determination of two $a b$ initio global six-dimensional potential energy surfaces, including the dissociation pathway, by Wei and Wyatt ${ }^{3}$ and by Yamamoto and Kato. ${ }^{4}$

\footnotetext{
${ }^{a)}$ Present address: Department of Chemistry, University of California, Berkeley, CA 94720-1460; electronic mail: viel@1sd.univ-montp2.fr

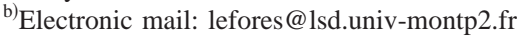

In this paper, we report the exact quantum calculation of the lowest 150 vibrational states of the HFCO molecule. The calculation and analysis of highly excited states of such a tetra-atomic molecule still represents a challenging task. Because the number of internal coordinates doubles as compared to triatomics, the size of the basis set roughly squares as a consequence. Such a huge increase in the dimension of the quantum system under study implies the revisiting of the successive steps entering the calculation.

The first point concerns the choice of the coordinate system used in order to represent the molecule. If valence coordinates appear to be a natural choice due to their physical meaning, they often lead to an intricate expression of the associated kinetic operator. ${ }^{5}$ Furthermore, this choice should be guided by their closest resemblance to the actual motions which take place in the energy regime considered. At low energy, it corresponds to curvilinear normal coordinates defined along the $G$ matrix formulation of Wilson. ${ }^{6}$ At high energy, especially close to the dissociation threshold, collision-type coordinates might lead to the best description. For that reason, we have chosen to use Jacobi coordinates to represent the HFCO molecule, as our final goal consists of the study of the dissociative states observed experimentally.

The second direction along which we have worked in this study concerns the contraction of the molecular basis set. Because of the very large bases to be handled, one has to resort to iterative methods in order to extract the energy levels of interest. It is well known that the convergence of such methods directly depends on the width of the spectrum associated with the Hamiltonian operator. ${ }^{7}$ The sequential adiabatic reduction (SAR) method of Bačic and Light has emerged as an optimal contraction scheme for the study of triatomic molecules ${ }^{8,9}$ and of Van der Waals complexes such as $(\mathrm{HCl})_{2} \cdot{ }^{10} \mathrm{In}$ its present formulation it, however, requires the ultimate storing of a full matrix whose dimension corresponds to the final contracted basis. We have used instead the related adiabatic pseudospectral (APS) method of 
Friesner et al. ${ }^{11}$ specifically designed in order to avoid this bottleneck. As a counterpart, one has to accept losing some of the contraction efficiency of the SAR method. However, for the system considered here, which has been shown to display a preferentially highly excited mode $\left(\nu_{6}\right)$, the APS method can be guided in order to tune the contraction scheme accordingly.

The last point we have particularly considered in this work deals with the assignment of energy levels coming out of the calculations. In the case of triatomic molecules, such an assignment is often made by visual inspection of the wave function plots. Such a procedure can hardly be envisioned when dealing with a tetra-atomic molecule due to the number of coordinates. In the present study, we have used and improved an automatic labeling procedure which has been previously proposed by one of us. ${ }^{12}$

The paper is organized as follows. Section II deals with the description of the HFCO molecular system, that is the coordinates used, the resulting Hamiltonian operator and its zero order approximation, and finally the two potential energy surfaces which have been considered. The primary molecular basis set and its contraction by means of the adiabatic pseudospectral method ${ }^{11}$ are described in Sec. III. Section IV presents the automatic procedure used for analyzing the energy levels and the resulting labels for the lowest 150 states. Finally, Sec. V concludes.

\section{DESCRIPTION OF THE HFCO MOLECULAR SYSTEM}

In this section, we first present the Jacobi coordinates which were used to describe the HFCO molecular system. We then give the resulting kinetic energy operator, in a compact algebraic form and in the associated differential form. From this last expression, we also define a zero-order approximation to be used for analysis later on. Finally, the two potential energy surfaces available for the HFCO molecule are briefly presented.

\section{A. Jacobi coordinates}

In a space-fixed (SF) frame attached to the center of mass of the molecule, three Jacobi vectors are needed in order to describe its shape and its orientation. We chose three vectors $\mathbf{O C}, \mathbf{g F}$, and $\mathbf{G H}$, as represented in Fig. 1, where $g$ and $G$ correspond to the OC and OCF centers of mass, respectively. For sake of simplicity, these vectors will be denoted $\mathbf{R}_{3}$ ( $\left.\equiv \mathbf{O C}\right), \mathbf{R}_{2}(\equiv \mathbf{g F})$, and $\mathbf{R}_{1}(\equiv \mathbf{G H})$. This choice of Jacobi vectors leads to a good correspondence with the valence coordinates as shown now.

The body-fixed (BF) frame has been defined by taking the $z$ axis parallel to the $\mathbf{R}_{3}$ vector, and choosing the $\mathbf{R}_{2}$ vector to lay in the upper $x G z$ half-plane $(x>0)$. The resulting six internal Jacobi coordinates correspond to the three vectors' lengths $R_{1}, R_{2}$, and $R_{3}$, the polar angles $\theta_{2}$ associated with $\mathbf{R}_{2}$, and the polar $\theta_{1}$ and azimuthal $\varphi$ angles associated with $\mathbf{R}_{1}$.

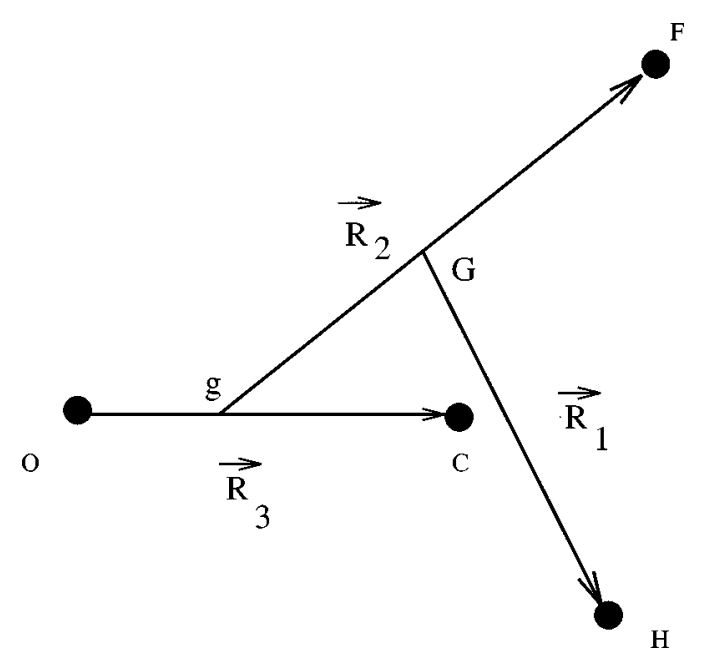

FIG. 1. Definition of the three Jacobi vectors $\mathbf{R}_{1}, \mathbf{R}_{2}$, and $\mathbf{R}_{3}$ used to describe the HFCO molecule.

\section{B. Algebraic kinetic energy operator}

In order to obtain the kinetic energy operator $\mathbf{T}$ in a very compact form we used the Gatti et al. vector formalism, ${ }^{13}$ which results in the expression

$$
\begin{aligned}
\mathbf{T}= & \frac{-\hbar^{2}}{2 \mu_{1}} \frac{\partial^{2}}{\partial R_{1}^{2}}-\frac{\hbar^{2}}{2 \mu_{2}} \frac{\partial^{2}}{\partial R_{2}^{2}}-\frac{\hbar^{2}}{2 \mu_{3}} \frac{\partial^{2}}{\partial R_{3}^{2}} \\
& +\left(\frac{1}{2 \mu_{1} R_{1}^{2}}+\frac{1}{2 \mu_{3} R_{3}^{2}}\right) \mathbf{L}_{1}^{\dagger} \cdot \mathbf{L}_{1}+\left(\frac{1}{2 \mu_{2} R_{2}^{2}}+\frac{1}{2 \mu_{3} R_{3}^{2}}\right) \mathbf{L}_{2}^{\dagger} \\
& \cdot \mathbf{L}_{2}+\frac{1}{2 \mu_{3} R_{3}^{2}}\left(\mathbf{L}_{1+} \mathbf{L}_{2-}+\mathbf{L}_{1-} \mathbf{L}_{2+}\right)+\frac{1}{\mu_{3} R_{3}^{2}} \mathbf{L}_{1 z} \mathbf{L}_{2 z} \\
& +\frac{1}{2 \mu_{3} R_{3}^{2}} \mathbf{J}^{\dagger} \cdot \mathbf{J}-\frac{1}{\mu_{3} R_{3}^{2}} \mathbf{J}_{z} \mathbf{J}_{z}-\frac{1}{2 \mu_{3} R_{3}^{2}}\left(\mathbf{J}_{+}\left[\mathbf{L}_{1-}+\mathbf{L}_{2-}\right]\right. \\
& \left.+\mathbf{J}_{-}\left[\mathbf{L}_{1+}+\mathbf{L}_{2+}\right]\right) .
\end{aligned}
$$

In the above formula, $\mathbf{L}_{i}$ stands for the angular momentum associated with the $\mathbf{R}_{i}$ vector, and $\mathbf{J}$ is the total angular momentum. $\mathbf{L}_{i \pm}$ and $\mathbf{J}_{ \pm}$correspond to the associated ladder operators. The volume element $d \mathrm{v}$ is given by $d \mathbf{v}$ $=d R_{1} d R_{2} d R_{3} \sin \theta_{1} d \theta_{1} \sin \theta_{2} d \theta_{2} d \varphi$, and the reduced masses $\mu$ 's have values given by

$$
\begin{aligned}
& \mu_{3}=\frac{m_{\mathrm{C}} \cdot m_{\mathrm{O}}}{m_{\mathrm{C}}+m_{\mathrm{O}}}, \\
& \mu_{2}=\frac{m_{\mathrm{F}} \cdot\left(m_{\mathrm{C}}+m_{\mathrm{O}}\right)}{m_{\mathrm{C}}+m_{\mathrm{O}}+m_{\mathrm{F}}}, \\
& \mu_{1}=\frac{m_{\mathrm{H}} \cdot\left(m_{\mathrm{C}}+m_{\mathrm{O}}+m_{\mathrm{F}}\right)}{m_{\mathrm{C}}+m_{\mathrm{O}}+m_{\mathrm{F}}+m_{\mathrm{H}}} .
\end{aligned}
$$

The total angular basis set which is naturally associated with this operator reads as

$$
\left\{P_{\ell_{1}}^{m_{1}}\left(\cos \theta_{1}\right) P_{\ell_{2}}^{\Omega-m_{2}}\left(\cos \theta_{2}\right) e^{i m_{1} \varphi} D_{M \Omega}^{J *}(\omega, \beta, \gamma)\right\},
$$

and leads to a simple analytical representation. In the above expression, $\Omega$ corresponds to the projection of the total an- 
gular momentum $\mathbf{J}$ onto the $z \mathrm{BF}$ axis, and $D_{M \Omega}^{J *}$ stands for the Wigner rotation function of the three Euler angles $\alpha, \beta$, and $\gamma$.

Although very compact, expression (2) cannot be directly used for a semirigid molecule such as HFCO. The reason comes from the fact that basis set (6) is delocalized over the whole angular configuration space $\theta_{1} \in[0, \pi] \otimes \theta_{2}$ $\in[0, \pi] \otimes \varphi \in[0,2 \pi]$. As will be shown in the next section, only a small part $(\sim 3 \%)$ of this space is actually sampled for excitation energies up to the dissociation limit into $\mathrm{CO}+\mathrm{HF}$. One then has to forgo this delocalized basis set and use instead a basis restricted to the energetically allowed angular space. This in turn implies switching to the differential form of the kinetic operator $\mathbf{T}$, as described now.

Using the expressions of the angular momentum operator $\mathbf{L}_{i}$ in the BF frame, ${ }^{13,14}$ one ends up with the new form, restricted here to the $J=0$ case,

$$
\begin{aligned}
\mathbf{T}^{J=0}= & \frac{-\hbar^{2}}{2 \mu_{1}} \frac{\partial^{2}}{\partial R_{1}^{2}}-\frac{\hbar^{2}}{2 \mu_{2}} \frac{\partial^{2}}{\partial R_{2}^{2}}-\frac{\hbar^{2}}{2 \mu_{3}} \frac{\partial^{2}}{\partial R_{3}^{2}}-\frac{\hbar^{2}}{2}\left[\frac{1}{\mu_{1} R_{1}^{2}}+\frac{1}{\mu_{3} R_{3}^{2}}\right]\left[\frac{1}{\sin \theta_{1}} \frac{\partial}{\partial \theta_{1}} \sin \theta_{1} \frac{\partial}{\partial \theta_{1}}+\frac{1}{\sin ^{2} \theta_{1}} \frac{\partial^{2}}{\partial \varphi^{2}}\right] \\
& -\frac{\hbar^{2}}{2}\left[\frac{1}{\mu_{2} R_{2}^{2}}+\frac{1}{\mu_{3} R_{3}^{2}}\right]\left[\frac{1}{\sin \theta_{2}} \frac{\partial}{\partial \theta_{2}} \sin \theta_{2} \frac{\partial}{\partial \theta_{2}}+\frac{1}{\sin ^{2} \theta_{2}} \frac{\partial^{2}}{\partial \varphi^{2}}\right] \\
& -\frac{\hbar^{2}}{\mu_{3} R_{3}^{2}}\left\{\cos \varphi \frac{\partial}{\partial \theta_{1}} \frac{\partial}{\partial \theta_{2}}-\left[\cot \theta_{2} \frac{\partial}{\partial \theta_{1}}+\cot \theta_{1} \frac{\partial}{\partial \theta_{2}}\right] \sin \varphi \frac{\partial}{\partial \varphi}\right\}+\frac{\hbar^{2}}{\mu_{3} R_{3}^{2}}\left[1+\cot \theta_{1} \cot \theta_{2} \cos \varphi\right] \frac{\partial^{2}}{\partial \varphi^{2}} .
\end{aligned}
$$

The last two terms of this new expression are, however, not Hermitian when considered individually. As noted by Carrington and Bramley, ${ }^{15}$ this feature precludes using the discrete variable representation approximation ${ }^{16}$ to handle them. Following their suggestion, these terms have been rewritten in an equivalent Hermitian form leading to the final expression for $\mathbf{T}$,

$$
\begin{aligned}
\mathbf{T}^{J=0}= & \frac{-\hbar^{2}}{2 \mu_{1}} \frac{\partial^{2}}{\partial R_{1}^{2}}-\frac{\hbar^{2}}{2 \mu_{2}} \frac{\partial^{2}}{\partial R_{2}^{2}}-\frac{\hbar^{2}}{2 \mu_{3}} \frac{\partial^{2}}{\partial R_{3}^{2}}-\frac{\hbar^{2}}{2}\left[\frac{1}{\mu_{1} R_{1}^{2}}+\frac{1}{\mu_{3} R_{3}^{2}}\right]\left[\frac{1}{\sin \theta_{1}} \frac{\partial}{\partial \theta_{1}} \sin \theta_{1} \frac{\partial}{\partial \theta_{1}}+\frac{1}{\sin ^{2} \theta_{1}} \frac{\partial^{2}}{\partial \varphi^{2}}\right] \\
& -\frac{\hbar^{2}}{2}\left[\frac{1}{\mu_{2} R_{2}^{2}}+\frac{1}{\mu_{3} R_{3}^{2}}\right]\left[\frac{1}{\sin \theta_{2}} \frac{\partial}{\partial \theta_{2}} \sin \theta_{2} \frac{\partial}{\partial \theta_{2}}+\frac{1}{\sin ^{2} \theta_{2}} \frac{\partial^{2}}{\partial \varphi^{2}}\right]-\frac{\hbar^{2}}{\mu_{3} R_{3}^{2}}\left\{-\frac{\partial^{2}}{\partial \varphi^{2}}-\cot \theta_{1} \cot \theta_{2}\left[\cos \varphi \frac{\partial^{2}}{\partial \varphi^{2}}-\sin \varphi \frac{\partial}{\partial \varphi}\right]\right. \\
& -\cot \theta_{2}\left[\frac{\partial}{\partial \theta_{1}}-\frac{1}{2} \cot \theta_{1}\right]\left[\sin \varphi \frac{\partial}{\partial \varphi}+\frac{1}{2} \cos \varphi\right]-\cot \theta_{1}\left[\frac{\partial}{\partial \theta_{2}}+\frac{1}{2} \cot \theta_{2}\right]\left[\sin \varphi \frac{\partial}{\partial \varphi}+\frac{1}{2} \cos \varphi\right] \\
& \left.+\cos \varphi\left[\frac{\partial}{\partial \theta_{1}}+\frac{1}{2} \cot \theta_{1}\right]\left[\frac{\partial}{\partial \theta_{2}}+\frac{1}{2} \cot \theta_{2}\right]+\frac{1}{4} \cot \theta_{1} \cot \theta_{2} \cos \varphi\right\} .
\end{aligned}
$$

\section{Zero-order Hamiltonian operator}

In order to perform the eigenstate analysis (Sec. IV), a zero-order description will be needed. We resorted to a normal mode approximation defined in the following way. Introducing the reduced coordinates

$$
\begin{aligned}
& r_{i}=R_{i}-R_{i_{\mathrm{eq}},} \\
& t_{i}=\cos \theta_{i}-\cos \theta_{i_{\mathrm{eq}}} \\
& f=\varphi-\varphi_{\mathrm{eq}},
\end{aligned}
$$

and setting the variables appearing in Eq. (7) to their equilibrium values, one ends up with the harmonic approximation

$$
\begin{aligned}
& \mathbf{H}^{0}=\sum_{i=1}^{3} \frac{-\hbar^{2}}{2 \mu_{i}} \frac{\partial^{2}}{\partial r_{i}^{2}}-\frac{\hbar^{2}}{2}\left[\frac{1}{\mu_{1} R_{1}^{2}}+\frac{1}{\mu_{3} R_{3}^{2}}\right]\left[\left(1-\cos ^{2} \theta_{1_{\mathrm{eq}}}\right) \frac{\partial^{2}}{\partial t_{1}^{2}}+\frac{1}{\sin ^{2} \theta_{1_{\mathrm{eq}}}} \frac{\partial^{2}}{\partial f^{2}}\right] \\
& -\frac{\hbar^{2}}{2}\left[\frac{1}{\mu_{2} R_{2}^{2}}+\frac{1}{\mu_{3} R_{3_{\mathrm{eq}}}^{2}}\right]\left[\left(1-\cos ^{2} \theta_{2_{\mathrm{eq}}}\right) \frac{\partial^{2}}{\partial t_{2}^{2}}+\frac{1}{\sin ^{2} \theta_{2_{\mathrm{eq}}}} \frac{\partial^{2}}{\partial f^{2}}\right]-\frac{\hbar^{2}}{\mu_{3} R_{3_{\mathrm{eq}}}^{2}} \sin \theta_{1} \sin \theta_{2_{\mathrm{eq}}} \cos \varphi_{\mathrm{eq}} \frac{\partial}{\partial t_{1}} \frac{\partial}{\partial t_{2}} \\
& -\frac{\hbar^{2}}{\mu_{3} R_{3_{\mathrm{eq}}}^{2}} \sin \varphi_{\mathrm{eq}}\left[\cot \theta_{2_{\mathrm{eq}}} \frac{\partial}{\partial t_{1}}+\cot \theta_{1_{\mathrm{eq}}} \frac{\partial}{\partial t_{2}}\right] \frac{\partial}{\partial f}+\frac{\hbar^{2}}{\mu_{3} R_{3_{\mathrm{eq}}}^{2}}\left[1+\cot \theta_{1_{\mathrm{eq}}} \cot \theta_{2_{\mathrm{eq}}} \cos \varphi_{\mathrm{eq}}\right] \frac{\partial^{2}}{\partial f^{2}}+V_{\mathrm{harm}}\left(r_{1}, r_{2}, r_{3}, t_{1}, t_{2}, f\right),
\end{aligned}
$$


TABLE I. Comparison of harmonic frequencies computed on the two potential surfaces with experiments, values being given in $\mathrm{cm}^{-1}$. The experimental values reported here correspond to transition frequencies as measured in the experiments. For each mode, an analysis in terms of the principal motion is given.

\begin{tabular}{lccc}
\hline \hline \multicolumn{1}{c}{ Normal mode } & Expt. frequencies $^{\mathrm{a}}$ & $\mathrm{WW}^{\mathrm{b}}$ & $\mathrm{YK}^{\mathrm{c}}$ \\
\hline$\nu_{1}(\mathrm{CH}$ stretch $)$ & 2981 & 3020 & 3141 \\
$\nu_{2}$ (CO stretch) & 1837 & 1808 & 1843 \\
$\nu_{3}$ (CH bend) & 1347 & 1389 & 1403 \\
$\nu_{4}$ (CF stretch) & 1065 & 1038 & 1074 \\
$\nu_{5}$ (FCO bend) & 662 & 631 & 670 \\
$\nu_{6}$ (out-of-plane H bend) & 1011 & 974 & 1038 \\
\hline \hline
\end{tabular}

${ }^{\mathrm{a}}$ Reference 2 .

${ }^{\mathrm{b}}$ Reference 3.

${ }^{\mathrm{c}}$ Reference 4.

associated with the volume element $d \tau$ $=d r_{1} d r_{2} d r_{3} d t_{1} d t_{2} d f$, and where $V_{\text {harm }}\left(r_{1}, r_{2}, r_{3}\right.$, $\left.t_{1}, t_{2}, f\right)$ corresponds to a quadratic expansion of the molecular potential around the equilibrium geometry.

Rewriting expression (10) under the general form

$$
\mathbf{H}^{0}=-\frac{\hbar^{2}}{2} \sum_{i j} T_{i j} \frac{\partial^{2}}{\partial q_{i} \partial q_{j}}+\frac{1}{2} \sum_{i j} V_{i j} q_{i} q_{j},
$$

one can then proceed along the Wilson $G$ matrix formulation, ${ }^{6}$ and define the normal modes $\left\{Q_{\alpha}\right\}$ in terms of the Jacobi coordinates

$$
Q_{\alpha}=\sum_{i} L_{i \alpha} q_{i}
$$

where the $\underline{\underline{L}}$ matrix is obtained from the generalized eigensystem

$$
\begin{aligned}
& \underline{\underline{V}} \cdot \underline{\underline{L}}=\underline{\underline{\underline{\lambda}}} \underline{\underline{T}}^{-1} \cdot \underline{\underline{L}}, \\
& \omega_{\alpha}=\sqrt{\lambda_{\alpha}} .
\end{aligned}
$$

\section{Potential energy surfaces}

Two, global, six-dimensional potential energy surfaces are available for the description of the HFCO molecule,

(i) using ab initio calculations at the MP4 level, Wei and Wyatt $^{3}$ have fitted a Simon-Parr-Finlan-type surface (WW) from 3855 geometries;

(ii) similarly, using about 4000 geometries computed at the restricted Hartree-Fock (RHF)/MP2 level, Yamamoto and $\mathrm{Kato}^{4}$ designed an analytical representation (YK) of the HFCO system, based on a partition of the configuration space into three different (equilibrium, transition state, and asymptotic) regions.

Both of these surfaces include the dissociation pathway into $\mathrm{HF}+\mathrm{CO}$.

Using the formulation introduced in Sec. IIC above [Eqs. (11) and (13)], we have computed the harmonic frequencies $\omega$ 's as given in Table I. Motions taking place in normal modes $\nu_{2}$ and $\nu_{6}$ are depicted in Fig. 2.
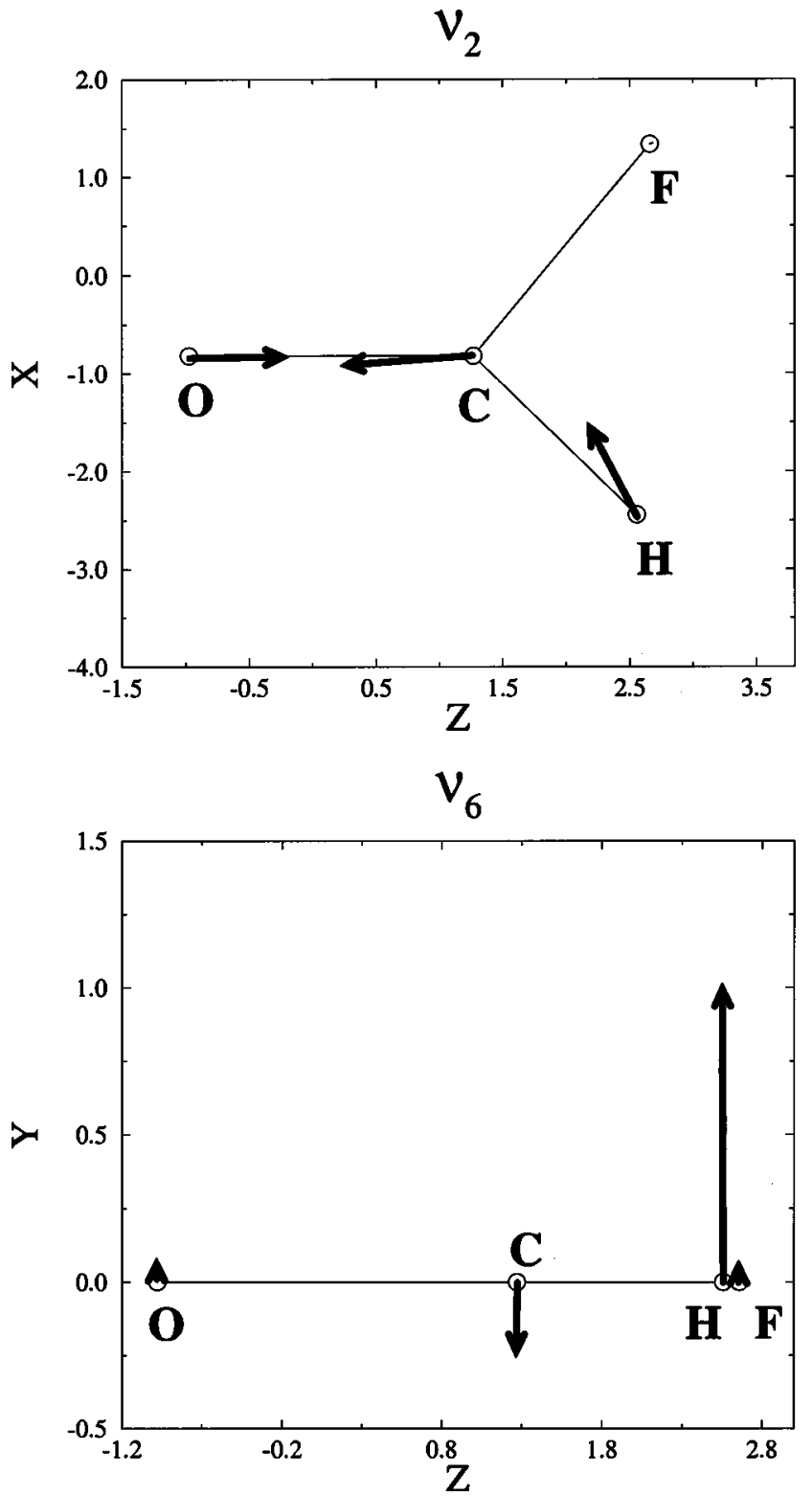

FIG. 2. Atomic displacements associated with normal modes $\nu_{2}$ and $\nu_{6}$, the molecule laying in the $x z$ plane.

\section{ADIABATIC CONTRACTION SCHEME}

In this section, we first describe the different discrete variable representations ${ }^{16}$ which were used in the primary (uncontracted) basis set. We then briefly recall the adiabatic pseudospectral (APS) method, ${ }^{11}$ and finally, we present its application to the HFCO molecule.

\section{A. Primary basis set}

The primary basis set consists of the direct product of a DVR along each coordinate

$$
\left\{\left|R_{1 a}\right\rangle\left|R_{2 b}\right\rangle\left|R_{3 c}\right\rangle\left|\theta_{1 \alpha}\right\rangle\left|\theta_{2 \beta}\right\rangle\left|\varphi_{\gamma}\right\rangle\right\}
$$

restricted to grid points where the potential energy is lower than some threshold $V_{\max }$,

$$
V\left(R_{1 a}, R_{2 b}, R_{3 c}, \theta_{1 \alpha}, \theta_{2 \beta}, \varphi_{\gamma}\right) \leqslant V_{\max } .
$$


For each radial coordinate, we used a sine based $\mathrm{DVR}^{17}$ associated with a uniform grid

$$
\left\{R_{p}=R_{<}+p \Delta R, \quad p=1, \ldots, N\right\},
$$

such that

$$
\begin{aligned}
& \left\langle R \mid R_{p}\right\rangle=\sum_{n=1}^{N} U_{p n} \sin \frac{n \pi\left(R-R_{<}\right)}{\left(R_{>}-R_{<}\right)}, \\
& U_{p n}=\sqrt{\frac{2}{N+1}} \sin \frac{n \pi\left(R p-R_{<}\right)}{\left(R_{>}-R_{<}\right)}, \\
& \left\langle R_{p}\left|-\frac{\partial^{2}}{\partial R^{2}}\right| R_{p^{\prime}}\right\rangle=\sum_{n=1}^{N} U_{p n}\left(\frac{n \pi}{R_{>}-R_{<}}\right)^{2} U_{n p^{\prime}} .
\end{aligned}
$$

Similarly, the azimuthal $\varphi$ angle was described by a sine based DVR $\left\{\left|\varphi_{\gamma}\right\rangle, \gamma=-Q, \ldots, Q\right\}$ associated with equidistant points, $\varphi_{\gamma}=\pi+\gamma \Delta \varphi$, distributed around the equilibrium value $\varphi_{0}=\pi$. No periodic boundary condition was enforced as the $\varphi=0$ and $2 \pi$ values are not energetically accessible below dissociation. The $C_{s}$ symmetry was taken into account by further defining two symmetry adapted DVR's,

$$
\begin{aligned}
& \left\{\left|\varphi_{\gamma}^{+}\right\rangle=\frac{1}{\sqrt{2\left(1+\delta_{0_{\gamma}}\right)}}\left\{\left|\varphi_{\gamma}\right\rangle+\left|\varphi_{\gamma}\right\rangle\right\}, \quad \gamma=0, \ldots, Q\right\}, \\
& \left\{\left|\varphi_{\gamma}^{-}\right\rangle=\frac{1}{\sqrt{2}}\left\{\left|\varphi_{\gamma}\right\rangle-\left|\varphi_{\bar{\gamma}}\right\rangle\right\}, \quad \gamma=1, \ldots, Q\right\} .
\end{aligned}
$$

The polar angles $\theta_{1}$ and $\theta_{2}$ were described by a GaussLegendre DVR ${ }^{16}$

$$
\left\{\left|\theta_{i}\right\rangle, i=1, \ldots, L\right\}
$$

i.e., corresponding to the abscissas of an $L$ points GaussLegendre quadrature

$$
\begin{aligned}
& \left\langle\theta \mid \theta_{i}\right\rangle=\sum_{\ell=0}^{L-1} U_{i \ell} \bar{P} \ell(\cos \theta), \\
& U_{i \ell}=\sqrt{\omega_{i}} \bar{P}\left(\cos \theta_{i}\right), \\
& \left\langle\theta_{i}\left|-\frac{1}{\sin \theta} \frac{\partial}{\partial \theta} \sin \theta \frac{\partial}{\partial \theta}\right| \theta_{i^{\prime}}\right\rangle=\sum_{\ell=0}^{L-1} U_{i \ell} \ell(\ell+1) U_{\ell i^{\prime}},
\end{aligned}
$$

where $\omega_{i}$ represents the weight associated with abscissa $\theta_{i}$ and $\bar{P} \ell$ a normalized Legendre polynomial.

Most of the calculations described later on were performed using a potential energy threshold $V_{\max }$ of $1.62 \mathrm{eV}$ for the definition of the primary grid. The resulting variation domain for the six coordinates is given in Table II for the WW surface as an example. Keeping only the points located below this energy threshold results in nondirect product $A^{\prime}$ and $A^{\prime \prime}$ grids of dimensions 595000 and 515000 , respectively. The restriction of the angular space with respect to total energy can be seen in Table II. Roughly, about 1/36 of the total angular space is sampled at energies below dissociation threshold.
TABLE II. Variation domain and number of posts used for each variable, corresponding to an energy threshold $V_{\max }$ of $1.62 \mathrm{eV}$. Values are given in bohrs and radians for distances and angles, respectively. The numbers in parentheses refer to the dimensions of the underlying Gauss-Legendre quadratures used for the polar angles $\theta_{1}$ and $\theta_{2}$. These data correspond to the WW surface (Ref. 3) but similar values were obtained for the YK surface (Ref. 4).

\begin{tabular}{ccc}
\hline \hline Variable & Variation domain & Number of points \\
\hline$R_{1}$ & {$[1.90,4.10]$} & 10 \\
$R_{2}$ & {$[2.83,4.51]$} & 27 \\
$R_{3}$ & {$[1.87,2.85]$} & 12 \\
$\theta_{1}$ & {$[0.48,1.54]$} & $18(50)$ \\
$\theta_{2}$ & {$[0.26,1.08]$} & $22(80)$ \\
$\varphi$ & {$[2.28,4.00]$} & 25 \\
\hline
\end{tabular}

Care must be taken when evaluating the matrix elements of kinetic angular terms in the DVR basis sets. Let us consider as an example the product

$$
\cot \theta_{2}\left(\frac{\partial}{\partial \theta_{1}}-\frac{1}{2} \cot \theta_{1}\right)\left(\sin \varphi \frac{\partial}{\partial \varphi}+\frac{1}{2} \cos \varphi\right)
$$

appearing in Eq. (8). It corresponds to the product of an Hermitian term $\cot \theta_{2}$ by two anti-Hermitian ones $\left(\partial / \partial \theta_{1}\right.$ $\left.-(1 / 2) \cot \theta_{1}\right)$ and $(\sin \varphi \partial / \partial \varphi+(1 / 2) \cos \varphi)$. Each of these last two terms will be anti-Hermitian provided its different components are evaluated in the same basis set. Accordingly, the two components $\sin \varphi \partial / \partial \varphi$ and $(1 / 2) \cos \varphi$ are first evaluated in the sine basis set, and their sum is then transformed to the DVR basis set by means of the associated collocation matrix $\underline{\underline{U}}$.

As a benchmark which will be used later on for testing the APS implementation, we computed the lowest 30 bound states in the primary DVR basis sets. Due to the very large size of these bases, we resorted to a Lanczos scheme, ${ }^{18}$

$$
\begin{aligned}
& \left|u_{n+1}\right\rangle \propto\left(\mathbf{H}-\alpha_{n}\right)\left|u_{n}\right\rangle-\beta_{n}\left|u_{n-1}\right\rangle, \\
& \alpha_{n}=\left\langle u_{n}|\mathbf{H}| u_{n}\right\rangle, \\
& \beta_{n+1}=\frac{1}{2}\left\{\left\langle u_{n+1}|\mathbf{H}| u_{n}\right\rangle+\left\langle u_{n}|\mathbf{H}| u_{n+1}\right\rangle\right\} .
\end{aligned}
$$

These levels are to be compared to those obtained within the APS formulation in Table IV.

\section{B. APS formulation}

The APS method, initially proposed by Friesner et al., ${ }^{11}$ has been used before for two tetra-atomic systems, namely the $6 \mathrm{D}$ hydrogen peroxide $\mathrm{H}_{2} \mathrm{O}_{2}{ }^{19}$ and the planar acetylene $\mathrm{C}_{2} \mathrm{H}_{2} .{ }^{20}$ We present below a general formulation which can be applied to any tetra-atomic system.

Let us consider a molecule described by six variables $\mathbf{q}$ $=\left(q_{1}, q_{2}, \ldots, q_{6}\right)$, ordered by increasing adiabaticity, and associated with the Hamiltonian operator

$$
\begin{aligned}
& \mathbf{H}=\mathbf{T}^{\text {diag }}+\mathbf{T}^{\text {off }}+V\left(q_{1}, q_{2}, \ldots, q_{6}\right), \\
& \mathbf{T}^{\text {diag }}=\sum_{n=1}^{N} f_{n}(\mathbf{q}) \mathbf{T}_{n}^{\text {diag }},
\end{aligned}
$$

where $\mathbf{T}^{\text {diag }}$ does not contain any cross derivative terms. 
TABLE III. Expansion of the normal modes in terms of the reduced Jacobi coordinates [Eqs. (12) and (13)]. These coefficients, calculated above for the YK surface, are very similar for the WW one.

\begin{tabular}{|c|c|c|c|c|c|c|}
\hline Mode & $\nu_{1}$ & $\nu_{2}$ & $\nu_{3}$ & $\nu_{4}$ & $\nu_{5}$ & $\nu_{6}$ \\
\hline & \multicolumn{6}{|c|}{ WW surface } \\
\hline \multirow[t]{2}{*}{ Frequencies $\left(\mathrm{cm}^{-1}\right)$} & 3020 & 1808 & 1389 & 1038 & 631 & 974 \\
\hline & \multicolumn{6}{|c|}{ YK surface } \\
\hline Frequencies & 3141 & 1843 & 1403 & 1074 & 670 & 1038 \\
\hline$r_{1}$ & .023 & .005 & .001 & .004 & .003 & .0 \\
\hline$r_{3}$ & -.002 & .009 & .000 & .000 & .0 & .0 \\
\hline$t_{1}$ & .0 & .0 & -.007 & .001 & .002 & .0 \\
\hline$r_{2}$ & .0 & .0 & -.001 & .006 & -.005 & .0 \\
\hline$t_{2}$ & .001 & .0 & .001 & -.002 & -.002 & .0 \\
\hline$f$ & .0 & .0 & .0 & .0 & .0 & .012 \\
\hline
\end{tabular}

The total wave function $\Psi(\mathbf{q})$ is expanded onto the sequential adiabatic basis set

$$
\begin{aligned}
\Psi\left(q_{1}, \ldots, q_{6}\right)= & \sum_{n_{1} \ldots n_{6}} \psi_{n_{1} \ldots n_{6}} \Phi_{n_{1}}\left(q_{1} ; q_{2}, \ldots, q_{6}\right) \\
& \times \Phi_{n_{2} n_{1}}\left(q_{2} ; q_{3}, \ldots, q_{6}\right) \times \cdots \times \Phi_{n_{6} \cdots n_{1}}\left(q_{6}\right),
\end{aligned}
$$

where

step 1

$$
\begin{array}{r}
\left\{f_{1}(\mathbf{q}) \mathbf{T}_{1}^{\mathrm{diag}}+V(\mathbf{q})\right\} \Phi_{n_{1}}\left(q_{1} ; q_{2}, \ldots, q_{6}\right) \\
=\varepsilon_{n_{1}}\left(q_{2} \ldots q_{6}\right) \Phi_{n_{1}}\left(q_{1} ; q_{2}, \ldots, q_{6}\right),
\end{array}
$$

step 2

$$
\begin{aligned}
\left\{\left\langle n_{1}\right|\right. & f_{2}(\mathbf{q})\left|n_{1}\right\rangle \mathbf{T}_{2}^{\text {diag }} \\
& \left.+\varepsilon_{n_{1}}\left(q_{2}, \ldots, q_{6}\right)\right\} \Phi_{n_{2} n_{1}}\left(q_{2} ; q_{3}, \ldots, q_{6}\right) \\
= & \varepsilon_{n_{2} n_{1}}\left(q_{3} \ldots q_{6}\right) \Phi_{n_{2} n_{1}}\left(q_{2} ; q_{3}, \ldots, q_{6}\right) \\
& \ldots . \ldots \ldots \ldots \ldots \ldots \ldots \ldots \ldots
\end{aligned}
$$

step 6

$$
\begin{aligned}
\left\{\left\langlen_{5} \cdots\right.\right. & \left.n_{1}\left|f_{5}(\mathbf{q})\right| n_{5} \cdots n_{1}\right\rangle \mathbf{T}_{6}^{\mathrm{diag}} \\
& \left.+\varepsilon_{n_{5} \cdots n_{1}}\left(q_{6}\right)\right\} \Phi_{n_{6} \cdots n_{1}}\left(q_{6}\right) \\
= & \varepsilon_{n_{6} \cdots n_{1}} \Phi_{n_{6} \cdots n_{1}}\left(q_{6}\right) .
\end{aligned}
$$

In practice, these different functions are computed at the nodes of a six-dimensional grid. In the following, we will use the letters $a b c x y z$ as indices for the grid points associated with variables $q_{1}, \ldots, q_{6}$, respectively. At each step, the new adiabatic basis set is truncated according to some energy criterion. For example, at step 3, only the $\Phi_{n_{3} n_{2} n_{1}}\left(q_{3} ; q_{4} \ldots q_{6}\right)$ states satisfying the inequality

$$
\varepsilon_{n_{3} n_{2} n_{1}}\left(q_{4 x}, q_{5 y}, q_{6 z}\right)-\min _{c x y z}\left\{\varepsilon_{n_{2} n_{1}}\left(q_{3 c}, q_{4 x}, q_{5 y}, q_{6 z}\right)\right\} \leqslant E_{\text {cut }}
$$

will be retained.
Once the adiabatic basis set [Eq. (33)] has been built, the Hamiltonian operator is acted onto $\Psi(\mathbf{q})$ in the following way. The wave function is sequentially expressed onto the six-dimensional grid

$$
\begin{aligned}
& \psi_{n_{1} \cdots n_{5} z}=\sum_{n_{6}} \mathcal{R}_{z n_{6}}^{\left(n_{5} \cdots n_{1}\right)} \psi_{n_{1} \cdots n_{6}}, \\
& \psi_{n_{1} \cdots n_{4} y z}=\sum_{n_{5}} \mathcal{R}_{y n_{5}}^{\left(n_{4} \cdots n_{1} z\right)} \psi_{n_{1} \cdots n_{5} z}, \\
& \cdots \cdots \cdots \cdots \cdots \cdots \cdots \cdots \cdots \cdots \\
& \psi_{a \cdots y z}=\sum_{n_{1}} \mathcal{R}_{a n_{1}}^{(b \cdots z)} \psi_{n_{1} b \cdots z} .
\end{aligned}
$$

The above collocation matrices $\mathcal{\mathcal { R }}$ correspond to the eigenvector matrices expressing the adiabatic states onto the grid. For step 3 mentioned before, it reads as

$$
\left|\Phi_{n_{3} n_{2} n_{1}}\left(q_{4 x} q_{5 y} q_{6 z}\right)\right\rangle=\sum_{c} \mathcal{R}_{c n_{3}}^{\left(n_{2} n_{1} x y z\right)}\left|q_{3 c}\right\rangle .
$$

Once the wave function is finally transformed to the $\left\{\psi_{a b c x y z}\right\}$ representation, one applies all the terms of the Hamiltonian operator, such as for example

$$
\begin{aligned}
& f_{2}(\mathbf{q})\left.\frac{\partial^{2}}{\partial q_{2}^{2}} \Psi(\mathbf{q})\right|_{a b c x y z} \\
& \quad=f_{2}\left(q_{1 a} \cdots q_{6 z}\right) \sum_{b^{\prime}}\left\langle q_{2 b}\left|\frac{\partial^{2}}{\partial q_{2}^{2}}\right| q_{2 b^{\prime}}\right\rangle \psi_{a b^{\prime} c x y z} .
\end{aligned}
$$

The cumulated result is then transformed back to the $\left\{\psi_{n_{1} \ldots n_{6}}\right\}$ contracted representation by means of the transposed collocations matrices $\mathcal{R}^{t}$.

It is clear from the above formulation that the APS basis set is essentially used as a filter. The Hamiltonian operator is actually acted in the primary grid representation, as in the straight DVR approach. But the result is filtered when transforming back to the adiabatic basis set, removing the high energy components.

\section{Application to HFCO}

The natural way to define the (increasing) adiabaticity hierarchy consists of considering normal modes by decreas- 
TABLE IV. Convergence of the energy levels (in $\mathrm{cm}^{-1}$ ) with respect to the cutoff $E_{\text {cut }}$ used during the construction of the APS basis set. $N_{\text {APS }}$ indicates the size of the basis, $E_{\mathrm{APS}}$, and $\Delta E_{\mathrm{APS}}$ the resulting energy and excitation energy, respectively. $E_{\mathrm{DVR}}$ makes reference to values obtained using the same primitive DVR basis set by means of a Lanczos scheme. $\mid \Delta E_{\mathrm{APS}}-$ $>D E_{\mathrm{DVR}} \mid$ gives the APS error on the excitation energy.

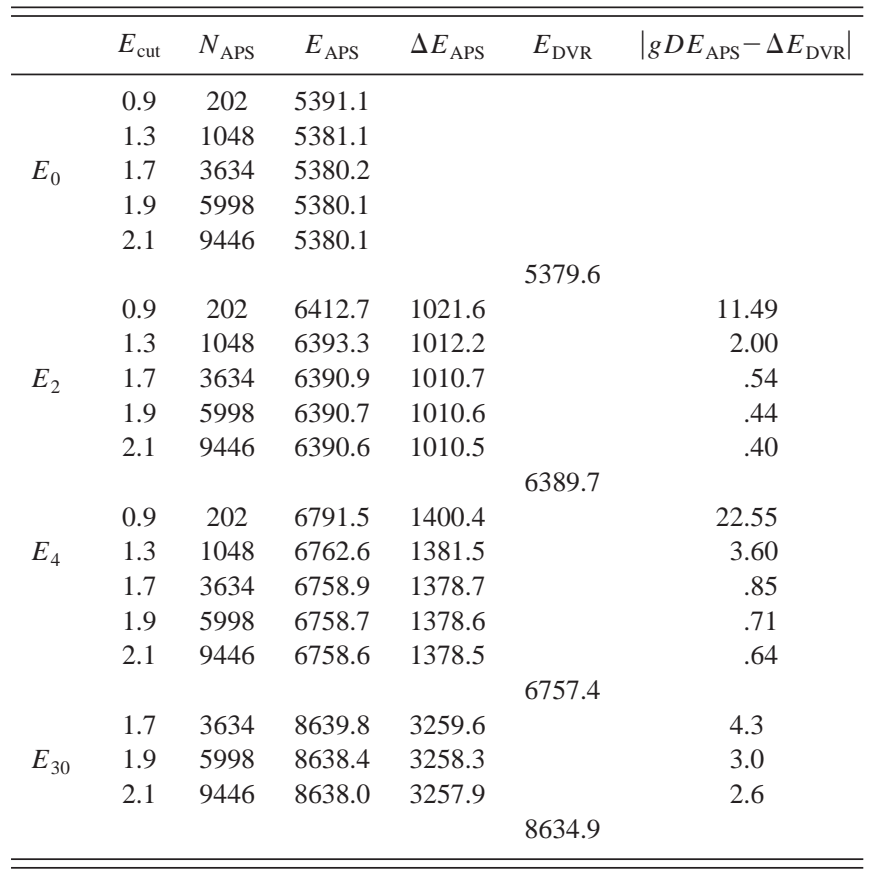

ing frequencies. However, as our preconditioning scheme relies on Jacobi coordinates rather than normal modes, we have to make the connection between these two sets of coordinates. Table III gives the expression of the normal modes in terms of reduced Jacobi coordinates [Eq. (9)]. The relative amplitudes allow us to make the following assignments: $\nu_{1}$ $\rightarrow R_{1}, \quad \nu_{2} \rightarrow R_{3}, \nu_{3} \rightarrow \theta_{1}, \nu_{4} \rightarrow R_{2}, \nu_{5} \rightarrow \theta_{2}, \nu_{6} \rightarrow \varphi$. We choose to define the adiabaticity ordering $R_{1}<R_{3}<\theta_{1}<R_{2}$ $<\theta_{2}<\varphi$ as the $\nu_{6}$ mode, associated with the $\varphi$ variable, was shown to be strongly decoupled at high energy.

In order to test the APS formulation as presented above, we performed a series of calculations of $A^{\prime \prime}$ levels on the WW surface, in which the energy cutoff $E_{\text {cut }}[\mathrm{Eq}$. (37)] has been systematically increased. In each calculation, the dimension $N_{\text {APS }}$ of the APS basis set is defined as the number of $\left(n_{1} n_{2} n_{3} n_{4} n_{5} n_{6}\right)$ sextets [Eq. (33)] finally retained at the end of the preconditioning procedure. These results are compared in Table IV to the Lanczos calculations performed in the primary DVR basis set, as described in Sec. III A. One can first note that, for the states presented in this table, a similar accuracy is achieved for cutoff values of $1.7 \mathrm{eV}$ or greater. This accuracy ranges from better than $1 \mathrm{~cm}^{-1}$ for the lowest states, to a few wave numbers for the 30 th $A^{\prime \prime}$ state. In the following, we used an energy cutoff value of $1.9 \mathrm{eV}$.

\section{RESULTS}

In this section, we first present the automatic labeling procedure which was used. The resulting energies and labels, as computed from the two potential surfaces, are then compared to the experimentally assigned energy levels.

\section{A. Automatic labeling procedure}

We used the method initially proposed by Menou and Leforestier in their study of the HCN molecule, ${ }^{12}$ and improved it in order to handle the increased complexity of a tetra-atomic molecule. Starting from the dispersion relation associated with the harmonic oscillator

$$
\left\langle\phi_{n}\left|Q^{2}-\bar{Q}^{2}\right| \phi_{n}\right\rangle \propto\left(n+\frac{1}{2}\right)
$$

one generates an initial guess $\mathbf{v}=\left(\mathrm{v}_{1}, \mathrm{v}_{2}, \ldots, \mathrm{v}_{6}\right)$ from the similar relations

$$
\begin{aligned}
& \left\langle\Psi_{N}\left|Q_{\alpha}^{2}-\bar{Q}_{\alpha}^{2}\right| \Psi_{N}\right\rangle=K_{\alpha}^{0}\left(\mathrm{v}_{\alpha}+\frac{1}{2}\right), \\
& K_{\alpha}^{0}=2\left\langle\Psi_{0}\left|Q_{\alpha}^{2}-\bar{Q}_{\alpha}^{2}\right| \Psi_{0}\right\rangle .
\end{aligned}
$$

In the above expression, $\Psi_{N}$ stands for an eigenstate, and $Q_{\alpha}$ corresponds to a normal mode [Eqs. (12) and (13)]. Due to the anharmonicity and intermode couplings, the labels $\mathbf{v}$ are no longer integers, but take real values. In order to match this set $\mathbf{v}$ to some set of labels $\mathbf{n}=\left(n_{1}, n_{2}, \ldots, n_{6}\right)$, two criteria are used:

(i) the first one consists of comparing the energy $E_{N}$ with those of levels in a waiting list. This list is initially composed of the six states with a single quantum of excitation, and is updated each time a new level $E_{N}$ has been assigned. The energies of the states in the waiting list are computed from the spectroscopical Hamiltonian,

$$
\begin{aligned}
E_{\mathrm{sp}}(\mathbf{n})= & \sum_{\alpha} \omega_{\alpha}\left(n_{\alpha}+\frac{1}{2}\right)+\sum_{\alpha \leqslant \beta} x_{\alpha \beta}\left(n_{\alpha}+\frac{1}{2}\right)\left(n_{\beta}+\frac{1}{2}\right) \\
& +\sum_{\alpha \leqslant \beta \leqslant \gamma} y_{\alpha \beta \gamma}\left(n_{\alpha}+\frac{1}{2}\right)\left(n_{\beta}+\frac{1}{2}\right)\left(n_{\gamma}+\frac{1}{2}\right) .
\end{aligned}
$$

In the above expression, only the frequencies $\omega$ 's are nonzero at the beginning of the labeling procedure, these values being taken as the normal frequencies. Each time a new level is assigned, all the $\omega$ 's, $x$ 's and $y$ 's parameters are updated by means of a least-square fit over all the already assigned levels. Waiting states with energies too far from the energy $E_{N}$ are not considered as candidates.

(ii) the second criterion is a similarity factor between the set $\mathbf{v}$ and each set $\mathbf{n}$ of the waiting list, defined as follows:

$$
\prod_{\alpha} \omega_{\alpha}^{-1 / 2} \exp \left[-\left(\mathrm{v}_{\alpha}-n_{\alpha}\right)^{2}\right] .
$$

This term is somewhat similar to a Franck-Condon factor between the two states $|\mathbf{v}\rangle$ and $|\mathbf{n}\rangle$.

These two criteria are then combined in a single factor,

$$
\eta=\exp \left\{-\sigma\left|E_{N}-E_{\mathrm{sp}}(\mathbf{n})\right|\right\} \prod_{\alpha} \omega_{\alpha}^{-1 / 2} \exp \left[-\left(\mathrm{v}_{\alpha}-n_{\alpha}\right)^{2}\right]
$$

where $\sigma$ is a constant to be adjusted (a value of $10^{-2} \mathrm{~cm}^{+1}$ has been used in our fit). Very often, the sole energy comparison between the waiting states and the level under study suffices to assign the label, as the first example shown in 
TABLE V. Examples of screening of the waiting list during the labeling procedure. The first example corresponds to a case where the energy $E_{N}$ of the state to be assigned exactly matches the predicted value $E_{s p}(\mathbf{n})$ of one of the states in the waiting list. The mismatch in energy of the second example is corrected for by the $\eta$ factor.

\begin{tabular}{|c|c|c|c|c|}
\hline$E_{N}\left(\mathrm{~cm}^{-1}\right)$ & v & $E_{\mathrm{sp}}(\mathbf{n})$ & $\mathbf{n}$ & $\eta$ \\
\hline \multirow[t]{8}{*}{8360.5} & 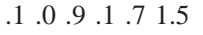 & & & \\
\hline & $\rightarrow$ & 8360.5 & 0001012 & $.3410^{-9}$ \\
\hline & & 8384.0 & $\begin{array}{llllll}0 & 0 & 2 & 0 & 2 & 0\end{array}$ & $.9010^{-11}$ \\
\hline & & 8403.7 & 100100 & $.1010^{-11}$ \\
\hline & & 8413.3 & $\begin{array}{llllll}0 & 0 & 1 & 2 & 1 & 0\end{array}$ & $.1710^{-11}$ \\
\hline & & 8435.6 & $\begin{array}{lllllll}0 & 1 & 0 & 1 & 2 & 0\end{array}$ & $.1610^{-12}$ \\
\hline & & 8453.5 & 000400 & $.1510^{-13}$ \\
\hline & & 8483.7 & 003000 & $.1110^{-12}$ \\
\hline \multirow[t]{9}{*}{8716.8} & $\begin{array}{l}.1 \\
1.5\end{array} .2 .2 .51 .4$ & & & \\
\hline & & 8718.5 & $\begin{array}{llllll}0 & 0 & 1 & 1 & 0 & 2\end{array}$ & $.6410^{-10}$ \\
\hline & $\rightarrow$ & 8727.6 & 010012 & $.1710^{-9}$ \\
\hline & & 8757.3 & 101000 & $.7110^{-11}$ \\
\hline & & 8765.7 & $\begin{array}{llllll}0 & 0 & 2 & 1 & 1 & 0\end{array}$ & $.2710^{-11}$ \\
\hline & & 8803.9 & 0111020 & $.2510^{-11}$ \\
\hline & & 8810.4 & 001300 & $.3910^{-12}$ \\
\hline & & 8823.1 & $\begin{array}{lllllll}0 & 1 & 0 & 2 & 1 & 0\end{array}$ & $.1410^{-11}$ \\
\hline & & 8835.7 & 000070 & $.1710^{-13}$ \\
\hline
\end{tabular}

Table V. The second example of the table corresponds to a case where the energy criterion does not lead to the right labels. The $\eta$ factor [Eq. (49)] allows one to single out the second state of the waiting list as the best candidate. It should be noted from this last example that the set $\mathbf{n}$ can be far from $\mathbf{v}$. The main reasons come from anharmonicity and intermode couplings.

\section{B. Calculated energy levels}

The APS formulation is basically designed to be used within an iterative scheme such as the Lanczos or the filter diagonalization method. ${ }^{21}$ The basic operation consists of acting the Hamiltonian operator $\mathbf{H}$ on a wave function expressed in the APS basis set [Eq. (33)]. In the study presented here, we were essentially interested in the low-lying levels in order to compare them to experimental results. For simplicity of the calculations, we chose to directly generate the Hamiltonian matrices (of $A^{\prime}$ and $A^{\prime \prime}$ symmetries) by applying $\mathbf{H}$ onto each element of the APS basis sets. The resulting matrices have been diagonalized by a direct method in order to obtain the 100 lowest states of each symmetry. Previous tests (see Table IV) have shown that most of the excitation energies should be converged within a few wave numbers for these levels.

Obtaining the $A^{\prime}$ and $A^{\prime \prime}$ levels as coming out from two different calculations renders the assignment easier. As $A^{\prime}$ and $A^{\prime \prime}$ states correspond to an even and odd number of quanta in the $\nu_{6}$ mode, respectively, this feature is used when screening the waiting list. It is important to remark that $A^{\prime}$ and $A^{\prime \prime}$ sets should be labeled at the same time: the spectroscopical Hamiltonian, which is used during the labeling procedure, can only be fitted if the two symmetries are handled together.

The lowest 150 energy levels have all been successfully labeled for the two surfaces. At the end of the fitting procedure, standard deviations between the calculated levels and
TABLE VI. Comparison of experimental levels (in $\mathrm{cm}^{-1}$ ) with those obtained from the YK and WW surfaces. All the assigned experimental levels are reported in this table. The lowest 150 levels have been computed for each surface. Standard deviations root-mean-square (rms) between the experimental and calculated levels are given at the bottom of the table.

\begin{tabular}{rccccccccc}
\hline \hline Expt. $^{\mathrm{a}}$ & YK & Number & WW & $n_{1}$ & $n_{2}$ & $n_{3}$ & $n_{4}$ & $n_{5}$ & $n_{6}$ \\
\hline 662.6 & 659.4 & 2 & 626.4 & 0 & 0 & 0 & 0 & 1 & 0 \\
1011.2 & 1020.5 & 3 & 968.8 & 0 & 0 & 0 & 0 & 0 & 1 \\
1064.9 & 1051.5 & 4 & 1017.8 & 0 & 0 & 0 & 1 & 0 & 0 \\
1324.1 & 1317.7 & 5 & 1255.1 & 0 & 0 & 0 & 0 & 2 & 0 \\
1342.3 & 1372.2 & 6 & 1371.1 & 0 & 0 & 1 & 0 & 0 & 0 \\
1719.3 & 1704.6 & 8 & 1639.5 & 0 & 0 & 0 & 1 & 1 & 0 \\
1836.8 & 1827.9 & 9 & 1770.5 & 0 & 1 & 0 & 0 & 0 & 0 \\
2115.6 & 2090.8 & 14 & 2029.2 & 0 & 0 & 0 & 2 & 0 & 0 \\
2412.0 & 2418.3 & 18 & 2376.1 & 0 & 0 & 1 & 1 & 0 & 0 \\
2494.2 & 2484.0 & 19 & 2393.4 & 0 & 1 & 0 & 0 & 1 & 0 \\
2841.0 & 2843.5 & 26 & 2727.5 & 0 & 1 & 0 & 0 & 0 & 1 \\
2895.0 & 2876.1 & 27 & 2787.1 & 0 & 1 & 0 & 1 & 0 & 0 \\
2981.2 & 3039.2 & 30 & 2974.4 & 1 & 0 & 0 & 0 & 0 & 0 \\
3150.6 & 3139.4 & 37 & 3016.2 & 0 & 1 & 0 & 0 & 2 & 0 \\
3652.8 & 3648.1 & 50 & 3526.7 & 0 & 2 & 0 & 0 & 0 & 0 \\
3838.1 & 3855.1 & 63 & 3686.9 & 0 & 1 & 0 & 0 & 0 & 2 \\
4302.9 & 4304.3 & 88 & 4138.3 & 0 & 2 & 0 & 0 & 1 & 0 \\
4307.5 & 4403.1 & 97 & 4335.9 & 1 & 0 & 1 & 0 & 0 & 0 \\
4493.9 & 4458.8 & 103 & 4323.5 & 0 & 0 & 1 & 1 & 0 & 2 \\
4653.1 & 4662.6 & 112 & 4474.4 & 0 & 2 & 0 & 0 & 0 & 1 \\
4705.2 & 4698.9 & 117 & 4546.4 & 0 & 2 & 0 & 1 & 0 & 0 \\
4817.6 & 4865.7 & 135 & 4649.9 & 0 & 1 & 0 & 0 & 0 & 3 \\
4955.0 & 4960.4 & 143 & 4758.8 & 0 & 2 & 0 & 0 & 2 & 0 \\
rms & 28 & & 92 & & & & & & \\
\hline \hline
\end{tabular}

${ }^{\mathrm{a}}$ Reference 22.

the associated spectroscopical Hamiltonian energies [Eq. (47)] displayed values of 1.85 and $1.18 \mathrm{~cm}^{-1}$ for the WW and YK surfaces, respectively. These deviations constitute an indicator of the accuracy of the assignment procedure. Table VI presents a comparison of the assigned experimental levels $^{22}$ with the calculated ones.

From this table, one can see that the WW surface tends to underestimate the $\nu_{2}, \nu_{4}, \nu_{5}$, and $\nu_{6}$ frequencies, while the $\nu_{1}$ and $\nu_{3}$ ones are overestimated. The $\nu_{1}$ frequency is pretty well described. By comparison to experimental results, this surface leads to a standard deviation of $92 \mathrm{~cm}^{-1}$.

Concerning the YK surface, the $\nu_{2}, \nu_{4}$, and $\nu_{5}$ frequencies are slightly underestimated, the $\nu_{6}$ slightly overestimated, while the $\nu_{1}$ and $\nu_{3}$ are more strongly overestimated. The corresponding standard deviation displays a value of 28 $\mathrm{cm}^{-1}$

\section{DISCUSSION}

In this study, we have presented an exact quantum sixdimensional calculation of the energy levels of the HFCO molecule. The choice of Jacobi vectors to describe the molecule has been shown to lead to a compact form of the Hamiltonian operator. Although such a formulation might not be optimum for the low-energy regime considered so far, it is totally general and applies as well above dissociation threshold. In that latter situation, other Jacobi vectors have to be redefined, but the Hamiltonian operator keeps exactly the same expression: only the reduced masses $\mu$ 's will change according to this new definition. 
An alternative approach, which has not been considered in this work, would consist of reframing the calculations in terms of the curvilinear normal modes introduced in Sec. III C. Such a set of coordinates would include in its definition part of the intermode couplings, kinetic as well as potential. In the low-energy regime, the associated normal basis set would constitute a quasioptimum description. This basis set could be even further improved by means of a self-consistent field (SCF) type preconditioning. ${ }^{23}$ As the zero-order Hamiltonian would correspond more closely to the exact one, smaller bases should result. Work along this direction is in progress.

The adiabatic pseudospectral method represents a basis set contraction procedure aimed at handling systems with a large number of variables. Although related to the sequential adiabatic reduction method of Bačić and Light, ${ }^{8,9}$ it differs from the latter in two main aspects:

at each reduction step, only the diagonal nonadiabatic correction terms are considered; while this feature impedes the contraction efficiency, it renders the bookkeeping task much easier.

(ii) as a consequence, the off-diagonal contribution has to be retrieved when acting the exact Hamiltonian operator on a wave function; this calculation is made particularly efficient by using a pseudospectral scheme, associated with the contracted basis set.

The APS method can be seen as a tradeoff between the highly optimized SAR approach, which would be difficult to implement in a six-dimensional problem such as HFCO, and a crude straight DVR description. It shares with the latter approach the feature to basically be a direct scheme: its application domain encompasses all iterative schemes which require the repetitive action of the Hamiltonian operator on some initial wave function. In particular, it is best suited to a filter diagonalization approach ${ }^{21}$ as a way to compute the highly excited states of tetra-atomic molecules.

The contraction scheme underlying the APS method allows it to be guided during the construction of the basis set. In particular, it is possible to retain states with only a few quanta in some given modes, while allowing more quanta in some other modes. Such a possibility could be exploited in the case of HFCO, due to experimental evidence of strong localization of energy in the $\nu_{6}$ mode.

This study has also shown that energy levels of a tetraatomic molecule can be successfully labeled by means of a fully automatic procedure. The key point in this method is the progressive fitting of a spectroscopical Hamiltonian during the labeling process. The resulting very small standard deviation between calculated and predicted energy levels gives confidence for extrapolating this Hamiltonian beyond the calculated levels, if necessary.

Finally, the calculations presented here serve as a test of the two HFCO potential energy surfaces, ${ }^{3,4}$ at least in the low-energy regime. The study presented so far has given some information concerning the anharmonicities and intermode couplings, i.e., beyond the normal mode approximation. Building such a surface is a tour de force, as the bound molecule, the transition state, and the outgoing products have to be described altogether. The comparison to experimental levels can be used for refining the existing surfaces.

\section{ACKNOWLEDGMENTS}

We would like to thank Professor Wyatt and Professor Kato for providing their potential energy routines, and Professor Moore for sending us data prior to publication. This work was partially supported by a CNRS-NSF grant, and by a grant of computer time from the Institut du Développement et des Ressources en Informatique Scientifique (IDRIS).

${ }^{1}$ Y. S. Choi, P. Teal, and C. B. Moore, J. Opt. Soc. Am. B 7, 1829 (1990).

${ }^{2}$ Y. S. Choi and C. B. Moore, J. Chem. Phys. 94, 5414 (1991).

${ }^{3}$ T. G. Wei and R. E. Wyatt, J. Phys. Chem. 97, 13580 (1993).

${ }^{4}$ T. Yamamoto and S. Kato, J. Chem. Phys. 107, 6114 (1997).

${ }^{5}$ M. J. Bramley and N. C. Handy, J. Chem. Phys. 98, 1378 (1993).

${ }^{6}$ E. B. Wilson, J. C. Decius, and P. C. Cross, Molecular Vibrations (McGraw-Hill, New York, 1955).

${ }^{7}$ R. Kosloff, in Dynamics of Molecules and Chemical Reactions, edited by R. E. Wyatt and J. Z. H. Zhang (Dekker, New York, 1996), p. 185.

${ }^{8}$ Z. Bačić and J. C. Light, J. Chem. Phys. 85, 4594 (1986).

${ }^{9}$ Z. Bačić and J. C. Light, J. Chem. Phys. 87, 4008 (1987)

${ }^{10}$ Y. Qiu and Z. Bačić, J. Chem. Phys. 106, 2158 (1997).

${ }^{11}$ R. A. Friesner, J. Bentley, M. Menou, and C. Leforestier, J. Chem. Phys. 99, 324 (1993).

${ }^{12}$ M. Menou and C. Leforestier, Chem. Phys. Lett. 210, 294 (1993).

${ }^{13}$ F. Gatti et al., J. Chem. Phys. 108, 8804 (1998).

${ }^{14}$ A. Viel, Ph.D. thesis, Université Montpellier, 1999.

${ }^{15}$ M. J. Bramley and T. Carrington, J. Chem. Phys. 99, 8519 (1993).

${ }^{16}$ J. C. Light, I. P. Hamilton, and J. V. Lill, J. Chem. Phys. 82, 1400 (1985).

${ }^{17}$ C. Leforestier, J. Chem. Phys. 94, 6388 (1991).

${ }^{18}$ C. Lanczos, J. Res. Natl. Bur. Stand. 45, 255 (1950).

${ }^{19}$ J. Antikainen, R. A. Friesner, and C. Leforestier, J. Chem. Phys. 102, 1270 (1995).

${ }^{20}$ J. Antikainen, Ph.D. thesis, Columbia University, 1995.

${ }^{21}$ D. Neuhauser, J. Chem. Phys. 93, 2611 (1990).

${ }^{22} \mathrm{C}$. B. Moore and R. Tadday, private communication.

${ }^{23}$ J. M. Bowman, Acc. Chem. Res. 19, 202 (1986) 